\title{
Cultivo de Ankistrodesmus gracilis (Chlorophyta) em laboratório à base de esterco suíno
}

\author{
Tatiana Betioli Fioresi \\ Lúcia Helena Sipaúba-Tavares* \\ Faculdade de Ciências Agrárias e Veterinárias, PG em Microbiologia, Centro de Aqüicultura, \\ Universidade Estadual Paulista-UNESP, CEP 14884-900, Jaboticabal, SP, Brasil \\ *Autora para correspondência \\ sipauba@caunesp.unesp.br
}

\section{Resumo}

O objetivo do presente trabalho foi testar a influência do meio à base de esterco suíno "in natura" e biodigerido, sobre o desenvolvimento, crescimento, comprimento total, peso seco e valor nutricional da microalga Ankistrodesmus gracilis. O pico de crescimento para A. gracilis foi maior no meio biodigerido $\left(6,2 \times 10^{7}\right.$ células. $\mathrm{mL}^{-1}$ ) no volume de $2 \mathrm{~L}$. Alta porcentagem de lipídio foi observada no meio "in natura", e, elevados teores de proteína no meio biodigerido em $2 \mathrm{~L}$. O biovolume, teor de cinzas e comprimento total foram diferentes $(\mathrm{p}<$ $0,05)$ entre os meios, o mesmo não ocorrendo para peso seco e fibra bruta $(\mathrm{p}>0,05)$. O requerimento de luz foi diferente entre os meios, com menor intensidade para o esterco biodigerido $\left(13,5 \mu \mathrm{E} \cdot \mathrm{cm}^{-2} \cdot \mathrm{s}^{-1}\right)$, indicando menor custo beneficio. O meio a base de esterco suíno, mostrou bons resultados para o crescimento de $A$. gracilis, com qualidade de água adequada para cultivo, podendo ser utilizado para cultura em larga escala.

Unitermos: alga, crescimento, composição bioquímica, esterco suíno, cultivo

\section{Abstract}

Ankistrodesmus gracilis (Chlorophyta) fertilized in swine manure in the laboratory. The objective of the present work was to investigate the influence of swine manure media on the growth, total length, dry weight, and nutritional value of Ankistrodesmus gracilis microalgae. Two media were measured: "in natura" and biodigested. The growth rate peak for $A$. gracilis was highest with biodigester treatment $\left(6.2 \times 10^{7}\right.$ cells. $\left.\mathrm{mL}^{-1}\right)$ on the $5^{\text {th }}$ day, at a volume of $2 \mathrm{~L}$. The highest percentage of lipids was verified for "in natura" media. Protein was highest $(\mathrm{p}>0.05)$ for the biodigested media at $2 \mathrm{~L}$. Biovolume, ash rate, and total length were different $(\mathrm{p}$ $<0.05)$ between treatments, but the same was not true for dry weight and crude fiber $(p>0.05)$. Light demand was also different between media, with lesser intensity being required for biodigested media $\left(13.5 \mu \mathrm{E} . \mathrm{cm}^{-2} . \mathrm{s}^{-}\right.$ $\left.{ }^{1}\right)$. In fact, the biodigested media proved to be cheaper in terms of cost and benefit. Generally, the medium containing swine manure, both "in natura" and biodigested, showed better results in A. gracilis development, with water quality adequate for culture systems. Swine manure in both forms may also be used in high-density cultures in the laboratory.

Key words: algae, growth, biochemical composition, swine manure, culture 


\section{Introdução}

O estudo do crescimento de microalgas é um aspecto importante para incrementar o conhecimento da biologia das diferentes espécies, favorecendo a produção em ambientes controlados. Atualmente, diferentes espécies de microalgas são produzidas em escala comercial resultando numa atividade de grande interesse pelo aporte do desenvolvimento de diferentes campos como a Aqüicultura e utilizadas como fonte de alimento para larvas de peixes, camarões e rãs (Sipaúba-Tavares e Rocha, 1993).

Em cultivos controlados de algas, os meios de cultura oferecem os nutrientes necessários para o crescimento ótimo de cada espécie, sendo o meio e técnica de cultivo a base para o estabelecimento da qualidade nutricional da alga.

Um dos principais problemas no cultivo em massa de microalgas utilizadas como alimento vivo, refere-se ao custo das substâncias químicas necessárias para a preparação dos meios de cultura. A redução do custo de produção é necessária e algumas pesquisas têm sido direcionadas neste sentido (Sipaúba-Tavares e Rocha, 1993; Adamsson, 2000; Hardy e Castro, 2000; Sipaúba-Tavares e Rocha, 2001).

O uso de esterco suíno como fonte alternativa para cultivo de microalgas em laboratório possui dois aspectos positivos: o primeiro é a grande disponibilidade do produto em virtude do crescimento da suinocultura no Brasil, ressalta-se que os dejetos produzidos neste seguimento contaminam mananciais e, o segundo aspecto, é a produção de um material de baixo custo, que propicie rápido crescimento e alto valor nutricional da alga cultivada.

Dentre as microalgas cultivadas, as clorofíceas têm sido amplamente utilizadas na alimentação de organismos aquáticos de água doce, particularmente Ankistrodesmus gracilis, mostrando-se bastante promissora como incremento da atividade aqüícola, sendo resistente ao manejo de cultivo e, selecionadas como alimento por larvas de peixes (Sipaúba-Tavares et al., 1999).

O conhecimento da biologia e a influência dos fatores tais como temperatura, luz, nutrientes, valor nutricional, são de grande importância no sucesso do cultivo de microalgas em sistemas controlados e que, posteriormente poderão servir direta ou indiretamente de alimento para larvas e alevinos de peixes.

Sendo assim, o objetivo deste estudo foi verificar a influência do meio a base de esterco suíno, "in natura" e biodigerido no crescimento de Ankistrodesmus gracilis, focalizando a biologia, valor nutricional e o efeito da qualidade da água de cultivo no desenvolvimento da alga em laboratório.

\section{Materiais e Métodos}

\section{Local e seleção da microalga Ankis- trodesmus gracilis}

A microalga Ankistrodesmus gracilis foi proveniente da Universidade Federal de São Carlos, linhagem $\mathrm{n}^{\circ} 005 \mathrm{CH}$, isolada da Represa do Broa (SP, Brasil) e, posteriormente, cultivada no Laboratório de Limnologia e Produção de Plâncton (Universidade Estadual Paulista-UNESP, Centro de Aqüicultura). O sistema de cultivo foi estático não axênico, com aeração constante, temperatura de $24,0 \pm 2,0^{\circ} \mathrm{C}$ sob iluminação de lâmpadas fluorescentes, com intensidade de 13,5 a $20,7 \mu \mathrm{E}$. $\mathrm{cm}^{-2} \cdot \mathrm{s}^{-1}$ no topo da cultura. Inicialmente, foi mantida em meio de cultura $\mathrm{CHU}_{12}$ e NPK (20:5:20) até sua aclimatação (Sipaúba-Tavares, 1995).

\section{Meio de Cultura}

O esterco suíno utilizado como meio de cultura foi cedido pelo Sistema de Produção de Suínos do Departamento de Zootecnia da UNESP (Jaboticabal-SP) em instalações de crescimento e terminação, compostas de baias de alvenaria com piso de concreto. O meio de cultura biodigerido foi obtido através do armazenamento de esterco suíno em biodigestores do tipo batelada onde ocorre o processo de fermentação, permanecendo armazenado por um período de 30 dias (Ortolani et al., 1991).

Foram utilizados $23,12 \mathrm{~g}$ do esterco suíno "in natura" (IN) e 80,8g do meio biodigerido em 30 dias (B30), ambos diluídos em $2.000 \mathrm{~mL}$ de água destilada e 
posteriormente, autoclavados. Após o resfriamento, foi retirada uma subamostra de $100 \mathrm{~mL}$, completada para $1.300 \mathrm{~mL}$ de água destilada, e mais $100 \mathrm{~mL}$ de inóculo de $A$. gracilis com densidade ao redor de $3 \times 10^{5}$ células. $\mathrm{mL}^{-1}$, para o cultivo em pequena escala (2L) (Figura 1). Quando o crescimento de A. gracilis atingiu a fase exponencial, foi transferido para volume maior (13L), totalizando 26L de inóculo para cada tanque de $250 \mathrm{~L}$, na densidade de $10 \times 10^{5}$ células.mL $\mathrm{m}^{-1}$ para o meio IN e de $7,1 \times 10^{6}$ células. $\mathrm{mL}^{-1}$ para B30. No meio B30, foi adicionado $0,01 \mathrm{~g} . \mathrm{L}^{-1}$ de vitaminas do complexo B (Sipaúba-Tavares e Bachion, 2002), com o objetivo de incrementar o crescimento da alga, cujos resultados não estavam sendo satisfatórios na ausência da vitamina. O experimento foi desenvolvido em triplicata (Figura 1).

\section{Crescimento}

Para o experimento com meio a base de esterco suíno, a alga foi mantida em intensidade de luz no topo da cultura de $20,7 \pm 0,2 \mu \mathrm{E} \cdot \mathrm{cm}^{-2} \cdot \mathrm{s}^{-1}$ para o meio IN e $13,5 \pm 1,2 \mu \mathrm{E} \cdot \mathrm{cm}^{-2} \cdot \mathrm{s}^{-1}$ para o B30, em ciclo de 24 horas. Para avaliar o crescimento de $A$. gracilis, alíquotas de $1 \mathrm{~mL}$ foram removidas diariamente, ao longo de 22 dias de cultivo e $2 \times 1 \mu \mathrm{L}$ de sub-amostras foram contados em hemocitômetro de Neubauer.

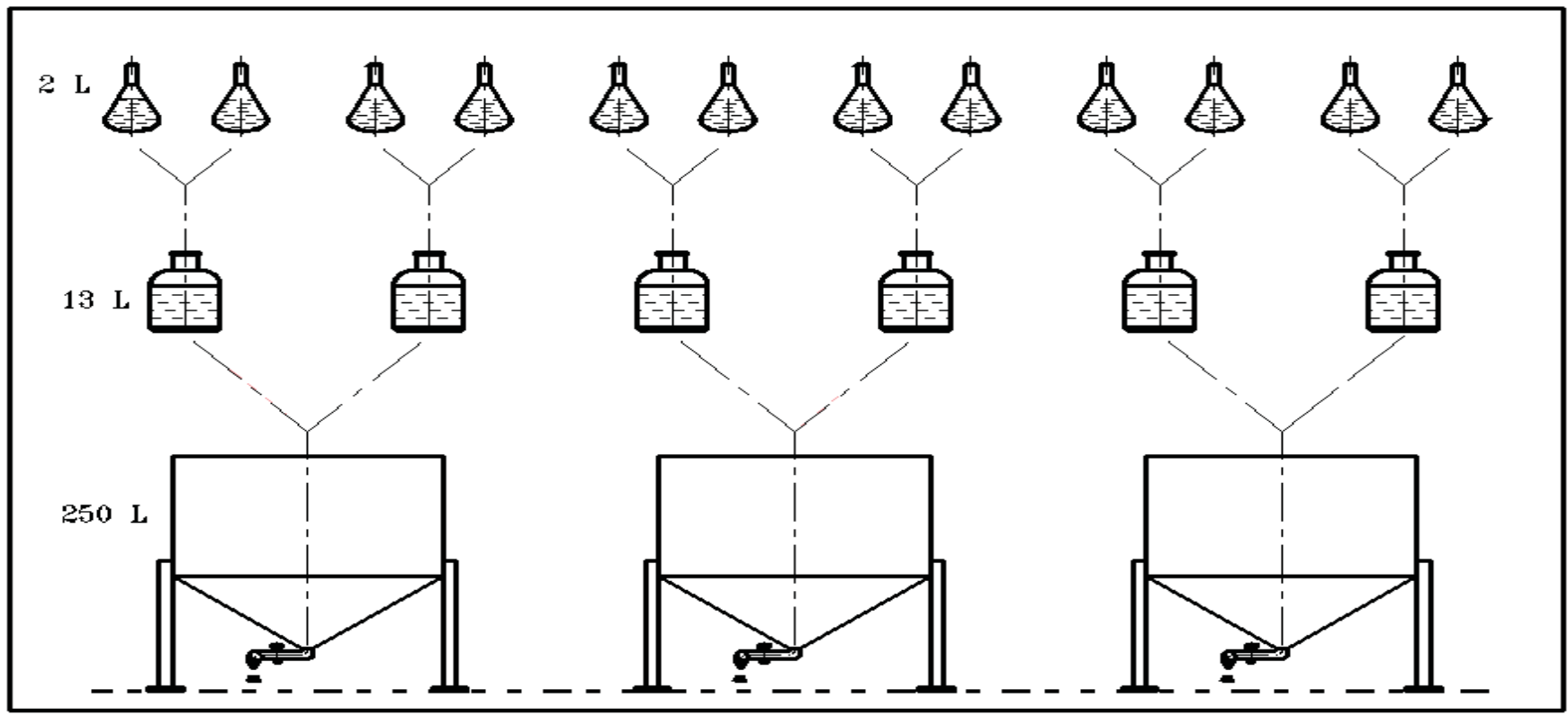

FIGURA 1: Esquema do cultivo da alga Ankistrodesmus gracilis em laboratório nos volumes de 2L, 13L e 250 L.

\section{Composição Química}

Ao final do experimento, ambos os volumes (2L e 250L) de $A$. gracilis foram concentrados em desnatadeira, liofilizado para análises de lipídeo, fibra e proteína seguindo a metodologia proposta por Association of Official Analytical Chemistry (1990). A composição química do meio de cultura também foi determinada de acordo com a metodologia descrita em Association of Official Analytical Chemistry (1990).

\section{Dados Biológicos}

O peso seco foi determinado obtendo-se $10 \mathrm{~mL}$ de cada réplica, sendo colhidas duas vezes por semana, com densidade média de 2,14 e 1,99 x $10^{7}$ células. $\mathrm{mL}^{-1}$ para IN e B30 em $2 \mathrm{~L}$ e no volume de $250 \mathrm{~L}$ de 6,6 e $6,1 \times 10^{6}$ células. $\mathrm{mL}^{-1}$ para IN e B30. As amostras foram filtradas em filtro de fibra de vidro (GFC $0,7 \mu \mathrm{m}$ de diâmetro de poro), previamente lavado em água destilada. Posteriormente, os filtros foram secos a $60^{\circ} \mathrm{C}$ e submetidos à pesagem, até peso constante. Para a determinação do conteúdo de cinzas da alga o ma- 
terial foi incinerado, em mufla a $500^{\circ} \mathrm{C}$, por 4 horas. O comprimento total $(\mu \mathrm{m})$ de 50 indivíduos de cada tratamento foi determinado em microscópio Olympus BX 50, com uso de sistema de análise de imagens ProPlus versão 4.1, Media Cybernetics, U.S.A. com ocular micrométrica em um aumento de 400x. O cálculo do biovolume foi realizado a partir das dimensões médias das células usando a forma geométrica mais apropriada que corresponde à fórmula de dois cones acoplados (Vollenweider, 1974, Bottrel et al., 1976).

\section{Análise Bacteriológica}

No meio de $2 \mathrm{~L}$, foram colhidas amostras da água de cultivo duas vezes por semana, totalizando sete amostragens, para cada meio de cultivo. Foi utilizada a técnica de tubos múltiplos para detecção de coliformes totais e coliformes fecais, sendo expressos em número mais provável (NMP.mL $\left.{ }^{-1}\right)$ e a técnica de plaqueamento em profundidade (pour plate) para microrganismos mesófilos expressos em unidade formadora de colônia (UFC. $\mathrm{mL}^{-1}$ ) (American Public Health Association, 1998).

\section{Características Hidrológicas}

Para acompanhamento da qualidade do meio de cultura, amostras da solução de água foram colhidas e analisadas três vezes por semana. As características físicas e químicas, como condutividade elétrica, $\mathrm{pH}$ e temperatura do meio, foram medidas utilizando aparelho digital Corning PS17, PS15 e PS16, respectivamente. O oxigênio dissolvido, carbono inorgânico e alcalinidade foram determinados segundo Golterman et al. (1978) e Mackereth et al. (1978). Amônia, nitrito, nitrato, fósforo total e ortofosfato foram determinados de acordo com Koroleff (1976) e Golterman et al. (1978). A clorofila- $a$ foi avaliada segundo Nush (1980) e a Demanda Bioquímica de Oxigênio $\left(\mathrm{DBO}_{5}\right)$, segundo metodologia descrita em American Public Health Association (1998).

\section{Análise Estatística}

Para a análise estatística, foi estabelecida a forma de um delineamento inteiramente casualizado, constituído por quatro tratamentos, com três repetições para cada um. Os dados foram comparados aplicando-se o teste de Tukey ao nível de 5\% de significância utilizando-se para isto o Sistema para Análises Estatísticas (V.2,0) - ESTAT.

\section{Resultados e Discussão}

Os dejetos de suínos apresentam uma considerável variação na sua composição, devido ao manejo nutricional adotado para os animais, porcentagem de diluição do dejeto, fase de vida que o animal se encontra e ainda, o tipo de tratamento empregado (Perdomo et al., 2001).

A composição química do meio de cultura à base de esterco suíno é mostrada na tabela 1 . O meio "in natura" (IN) apresentou maiores concentrações de nutrientes quando comparado ao meio biodigerido (B30), principalmente, em relação ao nitrogênio, cálcio e ferro, sendo que o inverso foi observado para o magnésio. Em geral, as menores concentrações dos nutrientes no B30, podem estar associadas ao fato de sofrer biodigestão anaeróbia, ou seja, primeiramente, os compostos orgânicos complexos são hidrolisados a compostos orgânicos simples (hidrólise) formando ácidos graxos de cadeia longa (acidogênese) e, acetato (acetogênese). Finalmente, ocorre a produção de metano (metanogênese) originando moléculas mais simples (Carvalho, 1998).

TABELA 1: Teor de nutrientes contidos no meio de esterco suíno para os meios "in natura" (IN) e biodigerido em 30 dias (B30).

\begin{tabular}{lcc}
\hline Composição & \multicolumn{2}{c}{ Meios } \\
\cline { 2 - 3 } Química & IN (mg.L $\left.\mathbf{L}^{-1}\right)$ & B30 (mg.L $\left.\mathbf{L}^{-1}\right)$ \\
\hline Nitrogênio & 10990 & 9380 \\
Fósforo & 6,89 & 4,18 \\
Potássio & 0,425 & 0,375 \\
Enxofre & 1,19 & 0,73 \\
Magnésio & 0,25 & 0,50 \\
Cálcio & 1100 & 500 \\
Manganês & 0,275 & 0,150 \\
Ferro & 1,575 & 0,600 \\
Zinco & 0,700 & 0,225 \\
Cobre & 0,900 & 0,275 \\
\hline
\end{tabular}

A luz é o fator essencial ao crescimento das algas, sem a qual as mesmas não podem processar suas ativi- 
dades metabólicas. O uso do esterco suíno para o cultivo de Ankistrodesmus gracilis, promoveu alta concentração de alga na cultura e baixo requerimento de luz. Segundo Brown et al. (1997) um dos maiores gastos na produção de algas em laboratório está relacionado à luz, cujas melhores condições estão entre 50 e $100 \mu \mathrm{E} . \mathrm{cm}^{-2} \mathrm{~s}^{-1}$, alternando de $12 \mathrm{em} 12$ horas. Neste estudo, a intensidade de luz foi em média de 13,0 a $20,7 \mu \mathrm{E} . \mathrm{cm}^{-2} \mathrm{~s}^{-1}$ no período de 24 horas, revelando resultado satisfatório na redução do custo de produção e rápido crescimento algal. No entanto, a baixa intensidade de luz utilizada neste estudo e o uso de tanques de cultivo de fibra de vidro translúcidos, para o volume de $250 \mathrm{~L}$, podem ter afetado o crescimento da alga quando comparados com os volumes de $2 \mathrm{~L}$, cujos cultivos foram realizados em frascos de vidro transparente promovendo maior biomassa (Figura 2).

Além disso, a diferença na intensidade luminosa pode estar relacionada ao fato de que o meio "in natu$r a$ " apresenta maior concentração de matéria orgânica tornando-o mais turvo e, no caso do meio biodigerido ocorre menor concentração de matéria orgânica, devido à ação de bactérias anaeróbias que fermentam este meio, tornando-o menos turvo facilitando a passagem de luz nos recipientes de cultivo.
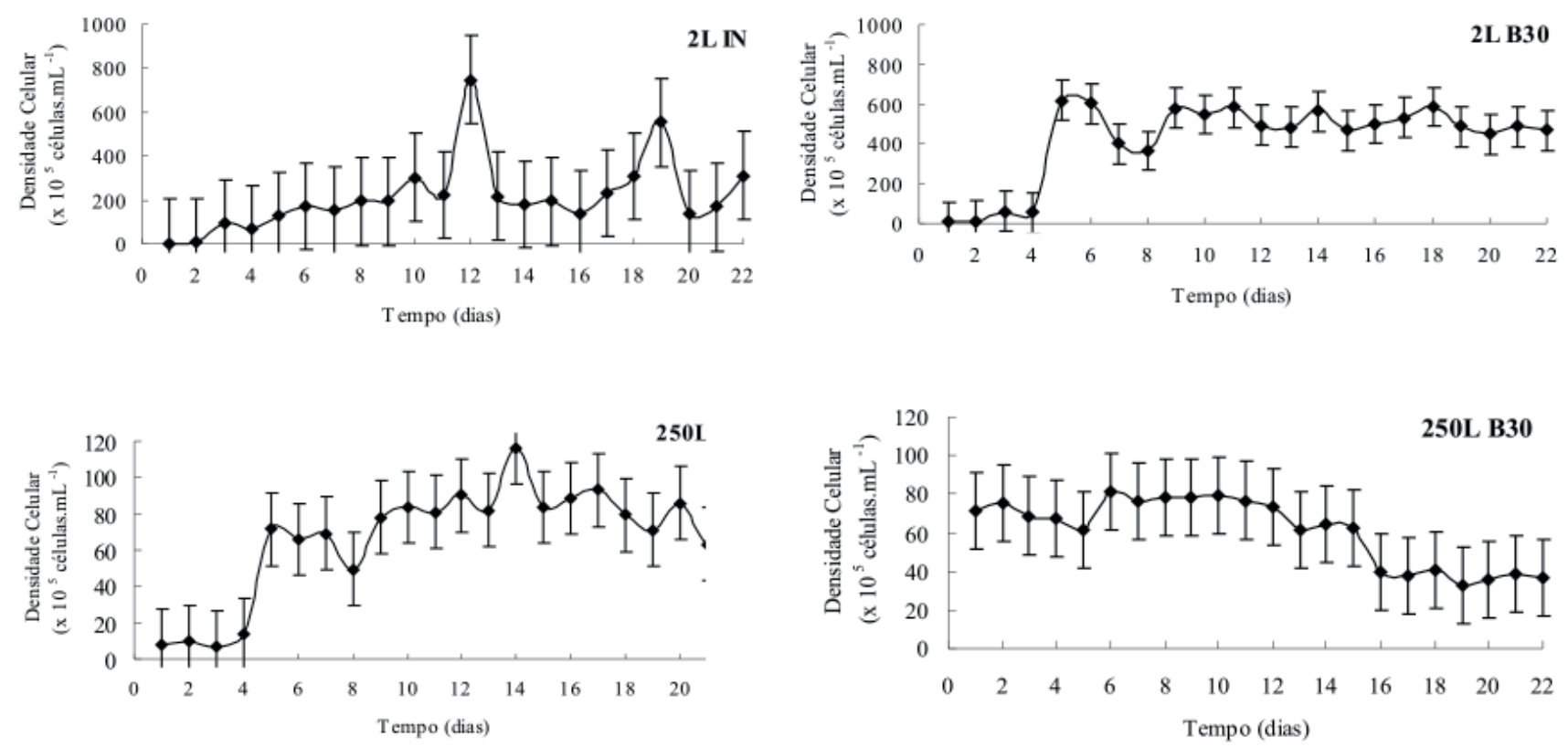

FIGURA 2: Crescimento da alga Ankistrodesmus gracilis cultivada em 2L e 250L em meios "in natura" (IN) e biodigerido em 30 dias (B30). 
Uma das características utilizadas para avaliação das algas como alimento é o tamanho, devido às grandes variações na forma e dimensões da célula, indicando o biovolume disponível como alimento (SipaúbaTavares e Rocha, 2001).

O biovolume (Figura 3) de A. gracilis em meio de esterco suíno variou de 70,33 a $427,86 \mu \mathrm{m}^{3}$ para o meio IN e 73,03 a $427,86 \mu \mathrm{m}^{3}$ para B30 apresentando diferenças significativas $(\mathrm{p}<0,05)$ entre os meios, com valores superiores aos encontrados por SipaúbaTavares e Rocha (1993), Sipaúba-Tavares et al. (1999) e Sipaúba-Tavares e Rocha (2001), porém similar ao obtido por Hardy e Castro (2000) utilizando meios tradicionais como $\mathrm{CHU}_{12}$ e NPK (20:5:20).

O valor nutricional de um organismo é de extrema importância em estudos de alimentação. A composição química bruta de uma espécie de microalga referente a aminoácidos, grau de insaturação de ácidos graxos ou conteúdo de vitaminas depende das condições de cultivo e do período de crescimento. No entanto, é possível generalizar a resposta de crescimento algal devido às alterações ambientais para as diferentes espécies (Brown et al., 1997).

A porção mineral da alga formada por cinzas variou de 4,21 a 7,53 pg.cél. ${ }^{-1}$ para o meio IN e de 3,2 a 3,36 pg.cél..$^{-1}$ para B30, sendo superior a obtida por
Sipaúba-Tavares e Rocha (1993) para a mesma alga cultivada em meio NPK $\left(0,01\right.$ pg.cél. $\left.{ }^{-1}\right)$ e inferiores ao obtido por Habib et al. (1997) para Chorella vulgaris $(18,6 \%)$ cultivada em efluente de moinho de óleo de palma (Tabela 2).

Os valores de proteína encontrados neste estudo estiveram acima de $30 \%$ de peso seco, com exceção do meio IN em 2L (19,3\%) (Tabela 2) sendo significativo $(\mathrm{p}<0,05)$ somente no meio B30 em 250L $(36,6 \%)$. Brown et al. (1997) também observaram níveis de proteína acima de $30 \%$ de peso seco atribuindo estes valores à aeração do meio.

O peso seco celular varia tanto em função da fonte de nitrogênio como nas fases de crescimento. No caso de $A$. gracilis as células cultivadas no meio IN em 2L foi mais elevado (85,0 pg.cel. $\left.{ }^{-1}\right)$ quando comparado com o B30 no mesmo volume ( 31 pg.cel..$\left.^{-1}\right)$, porém em larga escala (250L) o B30 $\left(70,8\right.$ pg.cel. $\left.{ }^{-1}\right)$ foi maior (Tabela 2).

Esses resultados foram superiores aos encontrados por Sipaúba-Tavares e Rocha (1993) trabalhando com $A$. gracilis utilizando o meio $\mathrm{CHU}_{12}\left(1,40\right.$ pg.célula $\left.{ }^{-1}\right)$. Em contra partida, Hardy e Castro (2000) utilizando meio de cultura NPK (20:5:20) para o cultivo de A. gracilis obtiveram resultados de peso seco superiores $(125,0 \pm 13,1$ pg.célula $\left.{ }^{-1}\right)$ aos encontrados neste estudo.

\section{$\square$ Biovolume $\rightarrow-$ Comprimento Total}

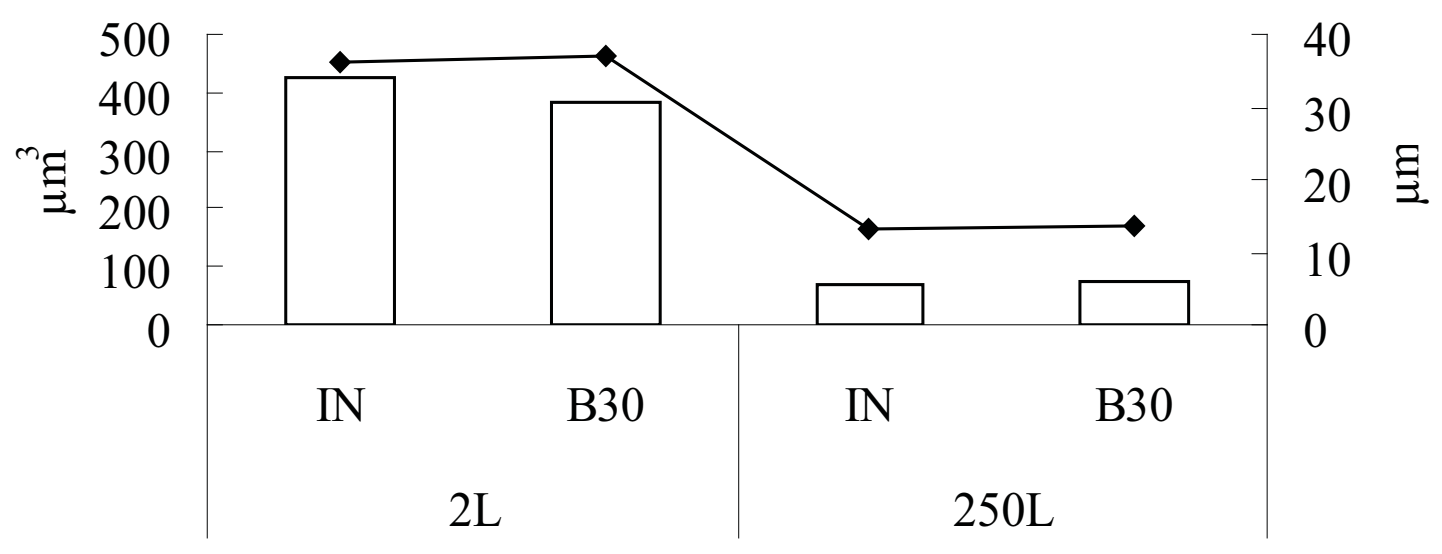

FIGURA 3: Biovolume e comprimento total da alga Ankistrodesmus gracilis cultivada em 2L e 250L nos meios "in natura" (IN) e biodigerido em 30 dias (B30). 
TABELA 2: Composição química da alga Ankistrodesmus gracilis cultivada em 2L e 250L nos meios "in natura" (IN) e biodigerido em 30 dias (B30).

\begin{tabular}{lcccc}
\hline \multirow{2}{*}{ Características } & 2L & \multicolumn{3}{c}{$\mathbf{2 5 0 L}$} \\
\cline { 2 - 5 } & $\mathbf{I N}$ & $\mathbf{B 3 0}$ & $\mathbf{I N}$ & $\mathbf{B 3 0}$ \\
\hline${\text { Cinzas (pg.cél. } .^{-1} \text { ) }}^{*}$ & $7,5 \pm 1,7^{\mathrm{a}}$ & $3,2 \pm 9,4^{\mathrm{a}}$ & $4,2 \pm 3,2^{\mathrm{b}}$ & $3,4 \pm 2,1^{\mathrm{b}}$ \\
Peso Seco (pg.cél. $\left.{ }^{-1}\right)$ & $85,0 \pm 1,5^{\mathrm{a}}$ & $31,0 \pm 4,5^{\mathrm{a}}$ & $24,6 \pm 3,4^{\mathrm{a}}$ & $70,8 \pm 4,3^{\mathrm{a}}$ \\
Fibra Bruta (\% de PS) & $15,7 \pm 0,9^{\mathrm{a}}$ & $14,3 \pm 0,9^{\mathrm{a}}$ & $21,4 \pm 4,3^{\mathrm{a}}$ & $12,0 \pm 8,2^{\mathrm{a}}$ \\
Proteína Bruta (\% de PS) & $19,3 \pm 0,5^{\mathrm{a}}$ & $30,2 \pm 0,9^{\mathrm{a}}$ & $33,7 \pm 2,7^{\mathrm{a}}$ & $36,6 \pm 1,7^{\mathrm{b}}$ \\
Lipídeo (\% de PS) & $17,0 \pm 0,7^{\mathrm{a}}$ & $20,5 \pm 2,0^{\mathrm{a}}$ & $7,9 \pm 1,3^{\mathrm{b}}$ & $8,5 \pm 1,0^{\mathrm{b}}$ \\
\hline
\end{tabular}

As letras a e b, indicadas em duas das colunas, não coincidentes na mesma linha, representam diferenças significativas de $\mathrm{p}<0,05$. PS $=$ Peso Seco.

O teor de fibra constitui o resíduo orgânico insolúvel geralmente considerado como carboidrato não disponível numa dieta ou alimento, apresentando valores acima de $12 \%$ (Tabela 2) sendo maiores do que os obtidos pela Food Agriculture Organization (1994) para Chlorella vulgaris $(8,0 \%)$.

Não foi observada a presença de coliformes fecais nos dois meios, sendo detectado coliformes totais na proporção de $6,0 \times 10^{2}$ e $5,1 \times 10^{2} \mathrm{NMP} . \mathrm{mL}^{-1}$ para IN e B30, respectivamente, ao final do experimento. Houve uma diferença considerável no número de unidade formadora de colônia entre o início e o final do experimento, sendo representadas pelos mesófilos com maior número de colônias no meio IN, apresentando uma variação de $1,9 \times 10^{2}$ a $8 \times 10^{3}$ UFC.mL $L^{-1}$ e no B30 de 6,9 x $10^{3}$ a $3,1 \times 10^{3}$ UFC.mL ${ }^{-1}$. Este aumento pode estar associado ao fato do uso de culturas não axênicas e por contaminação das condições do próprio laboratório.

Segundo Lucas-Junior (1994) o número mais provável de coliformes em dejetos de suínos é da ordem de $3,3 \times 10^{6} \cdot \mathrm{g}^{-1}$ (coliformes fecais) e $8,9 \times 10^{9} / \mathrm{g}$ (coliformes totais), sendo estes números superiores aos encontrados neste trabalho $\left(5,1\right.$ a $\left.6,0 \times 10^{-3} \cdot \mathrm{g}^{-1}\right)$.

A qualidade da água do meio de cultura é de fundamental importância no desenvolvimento e crescimento de organismos tais como temperatura, luz, oxigenação, entre outros. Foram observados altos valores de amô- nia, fósforo total e ortofosfato no meio, associados à composição do esterco suíno (Tabela 4).

Entre os diversos componentes do meio de cultura a fonte ou concentração de nitrogênio pode afetar o crescimento e composição bioquímica das microalgas em cultivo, atingindo diretamente a produtividade e composição bioquímica da biomassa obtida (Molina et al., 1991).

Estudos prévios (Becker, 1988; Fidalgo, 1995) mostraram algumas variações na composição bioquímica relacionando o aumento do teor de carboidratos $\mathrm{e}$ lipídios com a diminuição da proteína em meios de cultura com baixas concentrações de nitrogênio e fósforo. Neste estudo, as baixas concentrações de nitrogênio e fósforo observadas no volume de $250 \mathrm{~L}$ propiciaram teores de proteína mais elevados e de lipídio mais baixos, quando comparados ao volume de $2 \mathrm{~L}$, cujas concentrações de nitrogênio e fósforo foram extremamente elevadas (Tabelas 2 e 4 ).

Nayar et al. (1998), avaliando a qualidade nutricional de Chlorella vulgaris utilizada como alimento vivo na aqüicultura, verificaram níveis de proteína bruta e lipídeo ao redor de $12,2 \%$ e 5,4\% respectivamente, inferiores aos obtidos neste estudo (19,3 a 36,6 \% e 7,9 a 20,5\%) (Tabela 2).

Comparando as concentrações de amônia na composição do meio a base de esterco suíno, os resultados obtidos foram baixos, sendo o fator luz responsável pela disponibilidade deste componente. Em geral, $A$. gracilis em meio comercial $\mathrm{CHU}_{12}$ e alternativo NPK 
é cultivada ao redor de $21,48 \mu \mathrm{E} \cdot \mathrm{cm}^{-2} \cdot \mathrm{s}^{-1}$ (Sipaúba-Tavares et al., 1999). Com esterco suíno a intensidade foi reduzida a $13,5 \mu \mathrm{E} \cdot \mathrm{cm}^{-2} \cdot \mathrm{s}^{-1}$, pois rapidamente passava para a fase senescente quando colocada em intensidades mais elevadas. Neste caso, como o rendimento algal foi satisfatório e, sendo a luz um dos custos mais elevados no cultivo (Brown et al., 1997), o uso de esterco suíno como meio de cultura possui um ponto positivo em relação ao custo benefício da produção.

A aeração constante do meio promoveu altas concentrações de oxigênio dissolvido acima de $7 \mathrm{mg} . \mathrm{L}^{-1}$, favorecendo o processo de nitrificação, com ausência total de nitrito no volume de $2 \mathrm{~L}$. O contrário foi observado no volume de $250 \mathrm{~L}$ apresentando valores de 1,58 e $2,53 \mu \mathrm{g} . \mathrm{L}^{-1}$ no meio IN e B30, respectivamente. A condutividade elétrica não apresentou diferenças significativas $(p>0,05)$ entre os meios utilizados com valores acima de $59 \mu{\mathrm{S} . \mathrm{cm}^{-1}}^{-1}$ devido à presença de matéria orgânica no meio de esterco (Tabela 3 ).

Os valores das diferentes formas de carbono inorgânico apresentaram oscilações entre os tratamentos, sendo o bicarbonato a forma predominante. Foi observada uma relação entre $\mathrm{CO}_{2}$ livre e $\mathrm{pH}$, com menor concentração $\left(6,7 \mathrm{mg} \cdot \mathrm{L}^{-1}\right) \mathrm{em} \mathrm{pH}$ alcalino no meio IN em 2L (Tabela 3). Os valores de bicarbonato e $\mathrm{CO}_{2}$ livre foram inferiores aos encontrado por Carvalho (1998) no cultivo de A. gracilis com esterco de aves "in natura" e biodigerido em diferentes tempos de degradação.

A variação da concentração da clorofila- $a$ acompanhou a curva de crescimento de $A$. gracilis com a maior em 2L (7,1mg. $\left.\mathrm{L}^{-1}\right)$ e menor em 250L $\left(0,6 \mathrm{mg} . \mathrm{L}^{-1}\right)$ concentração no meio $\mathrm{B} 30$ (Tabela 3 ). $\mathrm{A} \mathrm{DBO}_{5}$ do meio foi relativamente baixa em função da aeração do meio e, baixas $\left(8,7\right.$ a $\left.11,4 \mathrm{mg} . \mathrm{L}^{-1}\right)$ concentrações de bactérias (Tabela 3).

$\mathrm{O}$ meio à base de esterco suíno, propiciou resultados satisfatórios em relação ao valor nutricional e crescimento de $A$. gracilis quando comparado a outros meios alternativos para cultivo dessa alga como: NPK (Sipaúba-Tavares e Rocha, 1993; Sipaúba-Tavares et al., 1999), urina humana (Adamssom, 2000), esterco de poedeiras (Carvalho, 1998), entre outros. A eficiência do esterco suíno como adubo no cultivo de plâncton em tanques externos já foi constatado por Santeiro e PintoCoelho (2000), Santeiro et al. (2006), porém o uso de meio de cultura à base deste produto para cultivo em larga escala de alga clorofícea em laboratório é uma possibilidade real de ser fornecido como alimento vivo de alta qualidade para larvas e alevinos de peixes.

TABELA 3: Características físicas e químicas dos meios de cultivo "in natura" (IN) e biodigerido em 30 dias (B30) para o crescimento de Ankistrodesmus gracilis.

\begin{tabular}{|c|c|c|c|c|}
\hline \multirow{2}{*}{$\begin{array}{c}\text { Características } \\
\text { Hidrológicas }\end{array}$} & \multicolumn{2}{|c|}{$2 \mathrm{~L}$} & \multicolumn{2}{|c|}{ 250L } \\
\hline & IN & B30 & IN & B30 \\
\hline Temperatura $\left({ }^{\circ} \mathrm{C}\right)$ & $23,0 \pm 2,3^{\mathrm{a}}$ & $21,1 \pm 1,1^{\mathrm{a}}$ & $19,2 \pm 2,3^{b}$ & $20,0 \pm 1,4^{b}$ \\
\hline $\mathrm{pH}$ & $7,2 \pm 0,2^{\mathrm{a}}$ & $6,8 \pm 0,5^{\mathrm{a}}$ & $6,3 \pm 0,3^{\mathrm{b}}$ & $6,9 \pm 0,3^{b}$ \\
\hline Condutividade elétrica $\left(\mu \mathrm{S} . \mathrm{cm}^{-1}\right)$ & $73,5 \pm 6,4^{\mathrm{a}}$ & $63,9 \pm 9,9^{\mathrm{a}}$ & $59,5 \pm 33,2^{\mathrm{a}}$ & $96,0 \pm 58,8^{a}$ \\
\hline Alcalinidade $\left(\mathrm{mg} \cdot \mathrm{L}^{-1}\right)$ & $43,8 \pm 4,4^{\mathrm{a}}$ & $34,5 \pm 8,2^{\mathrm{a}}$ & $34,4 \pm 4,2^{b}$ & $32,4 \pm 13,2^{b}$ \\
\hline $\mathrm{OD}\left(\mathrm{mg} \cdot \mathrm{L}^{-1}\right)$ & $7,2 \pm 0,7^{\mathrm{a}}$ & $7,0 \pm 1,7^{\mathrm{a}}$ & $10,2 \pm 2,9^{b}$ & $8,9 \pm 3,6^{b}$ \\
\hline Carbonato (mg. $\left.\mathrm{L}^{-1}\right)$ & $0,043 \pm 0,2^{\mathrm{a}}$ & $0,018 \pm 0,01^{b}$ & $0,004 \pm 0,02^{\mathrm{b}}$ & $0,02 \pm 0,01^{\mathrm{b}}$ \\
\hline Bicarbonato (mg. $\mathrm{L}^{-1}$ ) & $54,5 \pm 7,5^{a}$ & $42,0 \pm 9,9^{\mathrm{b}}$ & $41,9 \pm 5,2^{\mathrm{b}}$ & $39,5 \pm 16,1^{b}$ \\
\hline $\mathrm{CO}_{2}$ livre $\left(\mathrm{mg} \cdot \mathrm{L}^{-1}\right)$ & $6,7 \pm 3,4^{\mathrm{a}}$ & $14,9 \pm 21,5^{\mathrm{a}}$ & $42,6 \pm 26,9^{b}$ & $10,8 \pm 11,9^{\mathrm{a}}$ \\
\hline Nitrato $\left(\mu \mathrm{g} . \mathrm{L}^{-1}\right)$ & $6,0 \pm 5,2^{\mathrm{a}}$ & $4,8 \pm 2,1^{\mathrm{a}}$ & $19,6 \pm 1,6^{\mathrm{a}}$ & $45,3 \pm 76,6^{b}$ \\
\hline Nitrito $\left(\mu \mathrm{g} . \mathrm{L}^{-1}\right)$ & $* *$ & $* *$ & $1,58 \pm 1,66^{\mathrm{a}}$ & $2,53 \pm 2,32^{\mathrm{a}}$ \\
\hline Amônia $\left(\mu \mathrm{g} . \mathrm{L}^{-1}\right)$ & $410,0 \pm 298,4^{\mathrm{a}}$ & $231,7 \pm 102^{b}$ & $5,5 \pm 4,8^{\mathrm{a}}$ & $39,0 \pm 22,6^{a}$ \\
\hline Ortofosfato $\left(\mu \mathrm{g} . \mathrm{L}^{-1}\right)$ & $494,6 \pm 249,5^{\mathrm{a}}$ & $238,2 \pm 144,8^{\mathrm{a}}$ & $4,3 \pm 2,11^{\mathrm{b}}$ & $3,8 \pm 1,3^{b}$ \\
\hline Fósforo Total $\left(\mu \mathrm{g} \cdot \mathrm{L}^{-1}\right)$ & $507,5 \pm 213,1^{\mathrm{a}}$ & $146,9 \pm 148,8^{b}$ & $22,7 \pm 19,1^{\mathrm{a}}$ & $65,4 \pm 12,6^{a}$ \\
\hline $\mathrm{DBO}_{5}\left(\mathrm{mg} \cdot \mathrm{L}^{-1}\right)$ & $11,4 \pm 0,6^{\mathrm{a}}$ & $8,7 \pm 1,5^{\mathrm{b}}$ & $10,6 \pm 2,4^{\mathrm{a}}$ & $9,3 \pm 3,2^{\mathrm{b}}$ \\
\hline Clorofila-a (mg. $\left.\mathrm{L}^{-1}\right)$ & $3,9 \pm 5,7^{\mathrm{a}}$ & $7,1 \pm 6,2^{b}$ & $1,0 \pm 0,1^{\mathrm{a}}$ & $0,6 \pm 0,1^{\mathrm{a}}$ \\
\hline
\end{tabular}

** Não detectado pelo método. As letras minúsculas na mesma coluna apresentam diferenças significativas de $\mathrm{p}<0,05$. OD: Oxigênio Dissolvido e $\mathrm{DBO}_{5}$ : Demanda Bioquímica de Oxigênio. 
Os resultados mostraram que o esterco suíno pode ser utilizado como fonte alternativa de meio de cultura para A. gracilis, sendo que o esterco com 30 dias de fermentação proporcionou melhor crescimento da alga, em volume de $2 \mathrm{~L}$, demonstrando que o processo de biodigestão anaeróbia propiciou maior disponibilidade de nutrientes do resíduo para as algas. Volumes maiores $(\geq 250 \mathrm{~L})$ são utilizados na produção de alimento vivo para larvas e alevinos de peixes. Como o nosso objetivo era produzir alimento de alta qualidade em larga escala como aporte nutricional para larvas de peixes ou organismos zooplanctônicos, o meio utilizado obteve bons resultados no volume de $250 \mathrm{~L}$ principalmente o meio IN, em função de possuir maior quantidade de nutrientes do que o $\mathrm{B} 30$, porém os coliformes fecais e microrganismos mesófilos foram maiores no meio IN. As condições da água de cultivo no meio B30 em 250L apresentaram concentrações de amônia, nitrato e fósforo maiores que o meio IN com elevada condutividade elétrica, no entanto, o teor de clorofila- $a$ foi mais baixo devido ao decréscimo no crescimento e com isto disponibilizando os nutrientes para o meio.

O consumo de energia para o cultivo é um fator de alto empreendimento, no caso do meio a base de esterco suíno o requerimento de luz foi bem inferior ao meio usualmente utilizado neste procedimento (NPK). Assim, estudos devem ser averiguados para o desenvolvimento de uma tecnologia de produção em massa dessa alga, utilizando esterco suíno como subproduto e avaliando o custo-benefício deste empreendimento.

\section{Referências}

Adamsson, M. 2000. Potential use of human urine by greenhouse culturing of microalgae (Scenedesmus acuminatus), zooplankton (Daphnia magna) and tomatoes (Lycopersicon). Ecological Engineering, 16: 243-254.

Association of Official Analytical Chemistry - A.O.A.C. 1990. Official methods of analysis. $15^{\text {th }}$ ed. Arlington, Virginia, USA, 683pp.

American Public Health Association - APHA 1998. Standard methods for the examination of water and wastewater. $20^{\text {th }} \mathrm{ed}$ American Public Health Association, AWWA, WPCF, Washington:, USA, 1569pp.

Becker, E. W. 1988. : Micro-algal biotechnology. In: Borowitzka, M. A. \& Borowitzka, L. J. (eds). Micro-algae for human and animal consumption. University Press, Cambridge, England, p.222256.
Bottrel, H. H.; Duncan, A.; Gliwicz, Z. M.; Grygierek, E.; Herzig, A.; Hillbrich-Ilkowska, A.; Kurasawa, H.; Larsson, P.; Weglenska, T. 1976. A review of problems in zooplankton production studies. Norway Journal, 24: 419-456.

Brown, M. R.; Jeffrey, S. W.; Volkman, J. K.; Dustan, G. A. 1997. Nutritional properties of microalgae for marine culture. Aquaculture, 151: 315-331.

Carvalho, D. D. G. 1998. Avaliação da qualidade de água fertilizada com esterco de aves de postura in natura e tratado em biodigestores em diferentes fases de fermentação. Monografia, Universidade Estadual Paulista, Brasil, 112pp.

Food Agriculture Organization - F.A.O 1994. Fishery information, data and statistics service: aquaculture production. FAO Fisheries Circular, Rome, Italia, 216pp.

Fidalgo, P. 1995. Variabilidad bioquímica de microalgas marinas en cultivo en función de la fuente de nitrógeno. Tese de Doutorado, Universidade de La Coruña, Espanha, 217pp.

Golterman, H. L.; Clymo, R. S.; Ohnstad, M. A. 1978. Methods for physical and chemical analysis of freshwater. Blackwell Scientific Publication, London, 213pp.

Habib, M. A. B.; Yusoff, F. M.; Phang, S. M.; Ang, K. J.; Mohamed, S. 1997. Nutritional values of chironomid larvae grown in palm oil mill effluent and algal culture. Aquaculture, 158: 95-105.

Hardy, E. R.; Castro, J. G. D. 2000. Qualidade nutricional de três espécies de clorofíceas cultivadas em laboratório. Acta Amazônica, 30 (1): 39-47.

Koroleff, F. 1976. Determination of nutrients. In: Grassno, F. K. (ed.). Methods of seawater analysis. Verlag Cemie, Weinhein, New York, USA, p.117-181.

Lucas-Junior, J. 1994. Algumas considerações sobre o uso do estrume de suínos como substrato para três sistemas de biodigestores anaeróbios. Tese de Livre Docência, Universidade Estadual Paulista, Brasil, 137pp.

Mackereth, F. J. H.; Heron, J.; Talling, J. F. 1978. Water analysis: Some revised methods for limnologists. Titus Wilson \& Sons Ltda, Ambleside, England, 121pp.

Molina, E.; Sanchez, J. A.; Garcia, F.; Fernandez, J. M.; Acién, F. E. 1994. Effect of growth rate of eicosapentaenoic acid and docosahexaenoic acid content of Isochrysis galbana in chemostat culture. Applied Microbiology Biotechnology, 41: 23-27.

Nayar, S.; Hegde, S.; Rao, P. S.; Sudha, P. 1998. Live organisms as feed in aquaculture. INFOFISH International, 4: 36-40.

Nush, E. A. 1980. Comparison of different methods for chlorophyll and phaeopigments determination. Archiv für Hydrobiology, 14: 14-36.

Ortolani, A. F.; Benincasa, M.; Lucas-Junior, J. 1991. Biodigestores rurais modelos indiano, chinês e batelada. FUNEP, Jaboticabal, Brasil, 35pp.

Perdomo, C. C.; Lima, G. J. M. M.; Nones, K. 2001. Produção de suínos e meio ambiente. IX ${ }^{\mathbf{0}}$ Anais do Seminário Nacional de Desenvolvimento da Suinocultura, Gramado, Brasil, p.8-24.

Rodrigues, J. B. R.; Belli Filho, P. 2004. Eficiência da microalga Chlorella minutissima no tratamento de resíduos de suinocultura enriquecido com uréia. Biotemas, 17 (2): 7- 26.

Santeiro, R. M.; Pinto-Coelho, R. M. 2000. Efeitos de fertilização na biomassa e qualidade nutricional do zooplâncton utilizado para 
alimentação de alevinos na estação de hidrobiologia e piscicultura de Furnas, MG. Acta Scientiarum Biological Science, 22 (3): 707-716.

Santeiro, R. M.; Pinto-Coelho, R. M.; Sipaúba-Tavares, L. H. 2006. Diurnal variation of zooplankton biochemical composition and biomass in plankton production tanks. Acta Scientiarum Biological Science, 28 (2): 103-108.

Sipaúba-Tavares, L. H. 1995. Limnologia aplicada à aqüicultura. FUNEP/UNESP, Jaboticabal, Brasil, 70pp.

Sipaúba-Tavares, L. H.; Bachion, M. A. 2002. Population growth and development of two species of Cladocera, Moina micrura and Diaphanosoma birgei. Brazilian Journal Biology, 62 (4A): 701711.
Sipaúba-Tavares, L. H.; Pelicioli, L. C.; Olivera, A. 1999. Use of inorganic (NPK) and the $\mathrm{CHU}_{12}$ medium for cultivation of Ankistrodesmus gracilis in laboratory. Brazilian Journal of Ecology, 1: 10-15.

Sipaúba-Tavares, L. H.; Rocha, O. 1993. Cultivo em larga escala de organismos planctônicos para alimentação de larvas e alevinos de peixes: I - algas clorofíceas. Biotemas, 6 (1): 93-106.

Sipaúba-Tavares, L. H.; Rocha, O. 2001. Produção de plâncton (fitoplâncton e zooplâncton) para alimentação de organismos aquáticos. Rima Editora, São Carlos, Brasil, 106pp.

Vollenweider, R. A. 1974. A manual on the methods for measuring primary production in aquatic environments. Blackwell Scientific Publication, Oxford, USA, 225pp. 